\title{
DISEÑO Y EVALUACIÓN EXPERIMENTAL DE UN NUEVO DISPOSITIVO MECANICO PARA EL CONTROL DE POTENCIA DE PEQUEÑOS AEROGENERADORES EN REGIMEN DE SOBREVELOCIDAD DEL VIENTO
}

\author{
DESIGN AND EXPERIMENTAL EVALUATION OF A NEW \\ MECHANISM FOR POWER CONTROL OF SMALL WIND \\ TURBINES OPERATING IN OVERSPEED WIND \\ CONDITIONS
}

\author{
Salome Gonzáles Chávez ${ }^{1}$, William Urcuhuaranga Jesús ${ }^{2}$ \\ RESUMEN
}

\begin{abstract}
En este trabajo se realiza el diseño y pruebas de un nuevo dispositivo mecánico acoplado a la Cola-Veleta de un pequeño aerogenerador, tal que autorregula y controla la potencia instantánea de generación durante las sobrevelocidades de viento. La faja costera rural y zonas altoandinas del Perú, poseen gran potencial eólico, pero contrariamente se tienen nulos servicios eléctricos. En este escenario, la dotación de electricidad mediante microaerogeneradores constituye una alternativa estratégica. Una desventaja de los vientos característicos de las zonas de influencia es su alta variación de velocidades, cuyo efecto adverso en el aerogenerador es la destrucción prematura del bobinado del generador de imanes permanentes y rotura de palas. Las pruebas de regulación y control de potencia de aerogeneración se realizaron en el Túnel de Viento del Laboratorio de Energía de la Facultad de Ingeniería Mecánica de la UNI. Los resultados se dan en las curvas de potencia del conjunto aerogenerador de ensayo, donde se demuestra el control del crecimiento de la potencia generada, en el rango de velocidades de viento superiores a la condición nominal de diseño del aerogenerador. También se demuestra la confiabilidad del mecanismo cuando se somete a velocidades extremas de viento hasta alcanzar el bloqueo del aerogenerador, así como la inmediata recuperación continua de operación a medida que la velocidad de viento disminuye hacia condiciones nominales de trabajo.
\end{abstract}

Palabras clave.- Control de potencia, Pequeño aerogenerador, Sobrevelocidad de viento, Dispositivo pivote de cola, Confiabilidad.

\begin{abstract}
In this work we make the design and testing of a new mechanical element coupled to the vane of a small wind turbine, such that self-regulates and controls the instantaneous power generating wind during overspeed. The rural coast an andean highlands of Peru have high wind potential and on the contrary zero electrical services, in this scenario the provision of electricity by small wind turbines is a strategy solution. One disadvantage of the winds from these areas is its high speed variation, whose adverse effect on the turbine generator is the premature destruction magnet generator winding and turbine blades breakage. Tests were performed on the Wind Tunnel Energy Laboratory-Mechanical Engineering Faculty, Universidad Nacional de Ingeniería-Perú. The results are given in the power curves of the
\end{abstract}

${ }^{1}$ Doctor, Docente Investigador de la Facultad de Ingeniería Mecánica, Universidad Nacional de Ingeniería ${ }^{2}$ Investigador de la Universidad Nacional Mayor de San Marcos. 
wind turbine prototype. We also demonstrated the reliability of the mechanism when submitting to extreme wind speeds up to the blocking of the turbine generator, as well as the immediate recovery operation when the wind speed returns to nominal turbine generator working conditions.

Keywords.- Power control, Small wind turbine, Wind overspeed, Tail pivot mechanism, Reliability.

\section{INTRODUCCION}

\section{OBJETIVO Y FUNDAMENTACION DE LA HIPOTESIS}

Para garantizar la confiabilidad y vida útil de un sistema de aerogeneración de pequeña potencia, es necesario dotarle de dispositivos reguladores y controladores de la potencia eléctrica instantánea, en el rango de velocidades del viento donde superan las condiciones nominales de diseño del aerogenerador.

Cuando existe un aumento brusco de la velocidad del viento por encima de las condiciones de diseño de un sistema microaerogenerador (conformado por una turbina tripala en acople directo con un generador de imanes permanentemente), aumenta la velocidad de giro y consecuentemente incrementa la potencia (proporcional al cubo de la velocidad del viento). Esto trae como resultados efectos prematuros adversos como: destrucción de las palas, quemado de bobinas del generador de imanes permanentes, quemado de diodos, destrucción de las baterías, etc. [1].

El objetivo es entonces calcular y diseñar un dispositivo de control y regulación de potencia del aerogenerador, confiable, con autonomía para el bloqueo a velocidades extremas de viento, e inmediato restablecimiento de la generación eléctrica a velocidades dentro del rango de operación; esto bajo el principio de economía de construcción y operación.

Para ello es necesario calcular, diseñar y construir un prototipo, luego probar su comportamiento en un banco simulador de viento, hasta lograr la versión o ajuste definitivo del dispositivo.

\section{ANTECEDENTES}

Los dispositivos de regulación y control de potencia utilizados en aerogeneradores se pueden clasificar en dos tipos: activos y pasivos. Los activos actúan directamente en la variación del paso de las palas y requieren de mecanismos auxiliares de mayor costo, por lo que se ajustan mejor a grandes aerogeneradores [2].

Los pasivos actúan de manera indirecta sacando a la turbina fuera del frente de viento en sobrevelocidades, utilizando mecanismos diversos que se distinguen entre ellos en su rendimiento, costos, calidad de regulación y estabilidad; su utilización se adopta mejor a pequeños aerogeneradores [3 y 4].

Entre los mecanismos de regulación y control de potencia utilizados tradicionalmente en microaerogeneración se tienen: Dispositivo de aleta estabilizadora, palas con "Flaps" incorporados, palas con contrapesos y aerogeneradores basculantes [5 y 6].

Las desventajas comparativas de éstos, frente a la propuesta son: poseen inestabilidades en el control de potencia, altos costos debido a sus elementos auxiliares requeridos $\mathrm{y}$, baja confiabilidad de operación

\section{SISTEMA PROPUESTO DISPOSITIVO PIVOTE DE COLA}

$\mathrm{Su}$ principio de funcionamiento se basa en hacer que la cola-veleta gire gracias a un mecanismo soporte con pivoteo inclinado. Además el eje del generador debe estar desfasado con respecto al eje del poste de giro.

Tal excentricidad hace que a altas velocidades de viento el rotor venza el momento de inercia de la cola, lográndose en conjunto un bloqueo lento o salida del frente de viento del rotor-generador.

Cuando el viento baja su velocidad, el peso de la cola veleta hace regresar al rotor hacia el frente de las líneas de corriente de viento, permitiendo así la recuperación continua de generación de potencia, 
ver Fig. 1. Las ventajas del diseño propuesto frente a los mecanismos masificados son:

- Dispositivo compacto y sin mecanismos auxiliares.

- Estabilidad de operación. No se produce vibraciones del sistema.

- Construcción no dificultosa.

- Continuidad y autonomía de operación en todo rango de velocidades.

- Bajos costos de fabricación. Los costos de operación prácticamente son nulos, basta engrasar una vez al año al pivote.

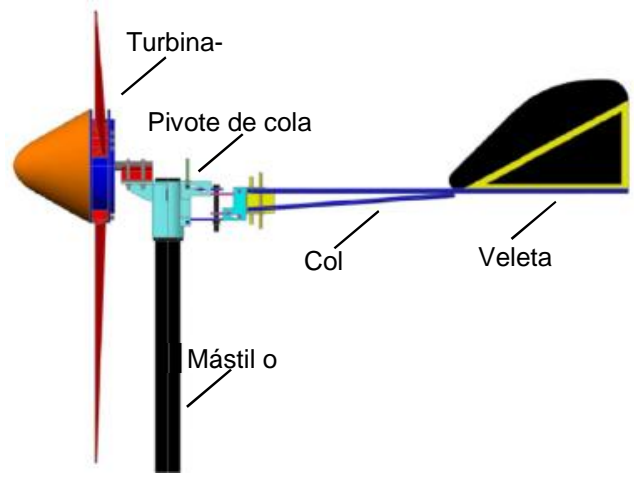

Fig. 1 Acople turbina-generador, mástil o torre, pivote de cola y veleta

Simbología:

$V: \quad$ Velocidad del viento

$\alpha$ : Angulo relativo entre la dirección del viento y el eje normal del rotor eólico, o ángulo de bloqueo

$\varphi: \quad \quad$ Angulo relativo entre el eje del rotor y el eje de la cola veleta

$\delta$ : $\quad$ Angulo horizontal entre el eje del rotor y el eje de la cola

$\psi: \quad \quad \quad \quad \quad \quad \quad \quad \quad \quad \quad \quad$ Angulo de incidencia del viento sobre la veleta (suma algebraica de $\alpha, \varphi$ y $\delta$ )

$\beta: \quad \quad$ Angulo vertical entre el eje del mástil y la bisagra o pivote

Lc : $\quad$ Longitud de la cola (distancia OA) (m)

Lcg : Distancia del punto "O" al centro de gravedad del conjunto cola-veleta (m)

$\mathrm{m}_{\mathrm{c}}$ : $\quad$ Masa de la cola con la veleta $(\mathrm{kg})$

$\rho: \quad$ Densidad del aire $\left(\mathrm{kg} / \mathrm{m}^{3}\right)$

Sc : $\quad$ Superficie de la veleta $\left(\mathrm{m}^{2}\right)$

S : $\quad$ Superficie abarcada por las palas $\left(\mathrm{m}^{2}\right)$

$\mathrm{g}$ : $\quad$ Aceleración de la gravedad $\left(\mathrm{m} / \mathrm{s}^{2}\right)$

$\overline{X B}^{0}$ : Vector distancia de $\mathrm{X}$ a $\mathrm{B}$ en la referencia $0(\mathrm{~m})$

$\overline{X O}$ : Vector distancia de $\mathrm{X}$ a $\mathrm{O}$ en la referencia $0(\mathrm{~m})$

$\overline{X C}_{G G}$ : Vector distancia de $\mathrm{X}$ a Cgg en la referencia $0(\mathrm{~m})$

e : $\quad$ Excentricidad del eje del rotor respecto del eje del poste (m)

$\bar{F}_{v, v e l, i}^{I I I}: \quad$ Componente i de la fuerza del viento sobre la veleta $(\mathrm{N})$ 
$C_{D, i}$ : Componente i del coeficiente de arrastre

$V_{i}^{I I I}: \quad$ Componente i del vector velocidad en la referencia III $(\mathrm{m} / \mathrm{s})$

$\bar{F}_{w, C, i}^{I I I}: \quad \quad$ Componente i del peso de la cola expresada en la referencia III (N)

$\bar{g}^{I I I}: \quad$ Vector gravedad en la referencia III $\left(\mathrm{m} / \mathrm{s}^{2}\right)$

$\mathrm{OA}^{\mathrm{III}}$ : Distancia OA en la referencia III (m)

$\mathrm{OC}_{\mathrm{GC}}^{\mathrm{III}}$ : $\quad$ Distancia $\mathrm{OC}_{\mathrm{GC}}$ en la referencia III (m)

$\bar{F}_{V, \text { rotor }, i}^{0}: \quad$ Componente i de la fuerza del viento sobre las palas (N)

$\mathrm{C}_{\mathrm{i}}$ : $\quad$ Coeficiente de empuje del viento sobre las palas

$V_{i}^{0}: \quad$ Componente i del vector velocidad en la referencia 0

$\bar{F}_{w, a, i}^{0}$ : Componente i del peso del aerogenerador expresado en la referencia $0(\mathrm{~N})$

$\mathrm{m}_{\mathrm{A}}$ : $\quad$ Masa del generador (con rotor eólico) $(\mathrm{kg})$

$\bar{M}_{\text {TOTAL }}^{\text {III }} \quad$ Momento total sobre el eje del poste de viraje (Nm)

$\bar{M}_{C O L A}^{0}$ : $\quad$ Momento resultante de la cola en la referencia $0(\mathrm{Nm})$

$\bar{M}_{C O L A}^{I I I}: \quad$ Momento resultante de la cola en la referencia III (Nm)

$\mathrm{X}$ : $\quad$ Punto donde se aplica el balance del sistema completo, en el eje del poste de viraje y a la misma altura que el punto $\mathrm{O}$

$\overline{X B}: \quad$ Vector distancia del punto $\mathrm{X}$ al $\mathrm{B}$ (centro del rotor eólico) (m)

$\overline{X C}_{G G}$ : Vector distancia del punto $\mathrm{X}$ al $\mathrm{C}_{\mathrm{GG}}$ (centro de gravedad del generador) (m)

$\overline{X O}$. Vector distancia del punto $\mathrm{X}$ al punto $\mathrm{O}(\mathrm{m})$

$\bar{F}_{C O L A}^{0}$ : Fuerza resultante de la cola en la referencia $0(\mathrm{Nm})$

$\mathrm{C}_{0-\mathrm{I}}$ : $\quad$ Matriz de cambio de base, de la referencia 0 a la referencia I

$\mathrm{C}_{\mathrm{I}-\mathrm{II}}$ : Matriz de cambio de base, de la referencia I a la referencia II

$\mathrm{C}_{\text {II-III }}$ : Matriz de cambio de base, de la referencia II a la referencia III

Mf : $\quad$ Momento que le resta la fricción al sistema

\section{DETERMINACION DEL SISTEMA DE REFERENCIA}

Primero se fija el sistema de coordenadas inicial, llamado base o referencia $0\left(\mathrm{X}_{0}, \mathrm{Y}_{0}, \mathrm{Z}_{0}\right)$, que corresponde al eje $\mathrm{Z}_{0}$ vertical, paralelo al poste $\mathrm{y}$ positivo hacia arriba, un eje $\mathrm{X}_{0}$ horizontal $\mathrm{y}$ paralelo al eje del rotor con sentido del viento $\mathrm{y}$, un eje $\mathrm{Y}_{0}$ horizontal y perpendicular al eje del rotor (ver figura 2).

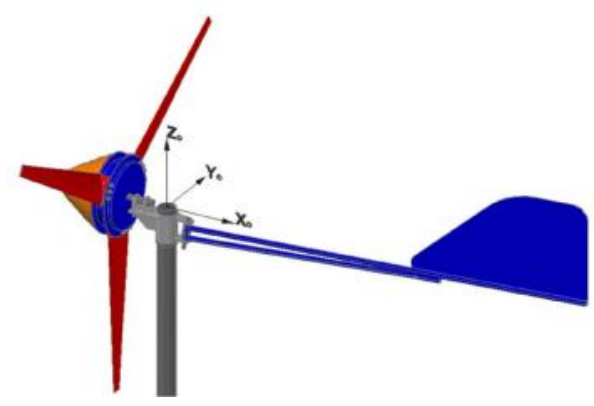

Fig. 2 Aerogenerador en la referencia 0, referida al rotor.
Referencia 0 a referencia I.- Se orienta el sistema de coordenadas de referencia 0 a fin de tener un eje $Y_{I}$ normal a la cola veleta, para ello se realiza un giro sobre el eje $Z_{0}$ de $\delta$ grados en el sentido positivo.

Al nuevo sistema de coordenadas se le llama referencia $\mathrm{I}\left(\mathrm{X}_{\mathrm{I}}, \mathrm{Y}_{\mathrm{I}}, \mathrm{Z}_{\mathrm{I}}\right)$.

Se fija la desviación $\delta$ para compensar el momento negativo que se genera en el sistema debido a la excentricidad "e" del rotor (ver figura 3 ).

Es decir, el objetivo del sistema de cola-veleta es mantener el rotor frontal al viento hasta una determinada velocidad, pero efecto de la excentricidad el rotor podría bloquearse a bajas velocidades de viento.

Para evitar ese bloqueo temprano, se desvía a la cola un ángulo $\delta$ a fin de que el viento ejerza una fuerza normal en la veleta y compense el momento negativo del sistema. 


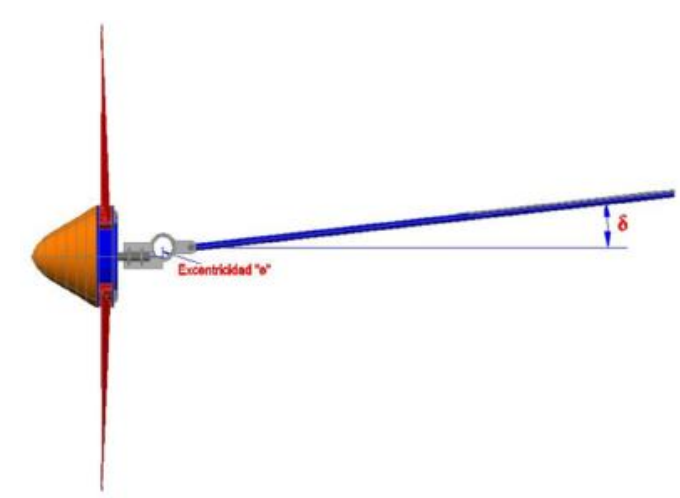

Fig. 3 Posición rotor-cola veleta en la referencia I.

La matriz de cambio de referencia 0 a la referencia I será:

$$
C_{0-I}=\left[\begin{array}{lcc}
\cos \delta & \operatorname{sen} \delta & 0 \\
-\operatorname{sen} \delta & \cos \delta & 0 \\
0 & 0 & 1
\end{array}\right]
$$

Referencia I a referencia II.- Al sistema de coordenadas de referencia I se realiza un segundo giro sobre $Y_{I}$ de $\beta$ grados positivo, para tener un $\mathrm{Z}_{\text {II }}$ paralelo al eje del pivote de cola, generándose así el nuevo sistema de coordenadas llamado referencia II $\left(\mathrm{X}_{\mathrm{II}}, \mathrm{Y}_{\mathrm{II}}, \mathrm{Z}_{\mathrm{II}}\right)$. Esta orientación se realiza porque la cola va a pivotear sobre un ángulo $\beta$, cuando el rotor inicie el bloqueo, fig. 4.

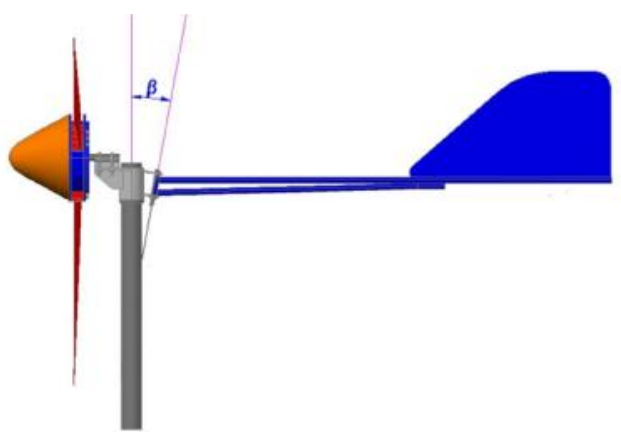

Fig. 4 Posición rotor-pivote de cola en la referencia II.

La matriz de cambio de referencia I a referencia II será:

$$
C_{I-I I}=\left[\begin{array}{ccc}
\cos \beta & 0 & -\operatorname{sen} \beta \\
0 & 1 & 0 \\
\operatorname{sen} \beta & 0 & \cos \beta
\end{array}\right]
$$

Referencia II a referencia III.- Al sistema de coordenadas de referencia II se realiza un tercer giro sobre $\mathrm{Z}_{\text {II }}$ de $\varphi$ grados negativos, generándose así un sistema de coordenadas de referencia III. Este giro se genera porque la cola debido al pivote (paralelo al eje $\mathrm{Z}_{\mathrm{II}}$ ) se mueve un ángulo $\varphi$ respecto a su posición original cuando el rotor comienza a bloquearse, fig. 5 .

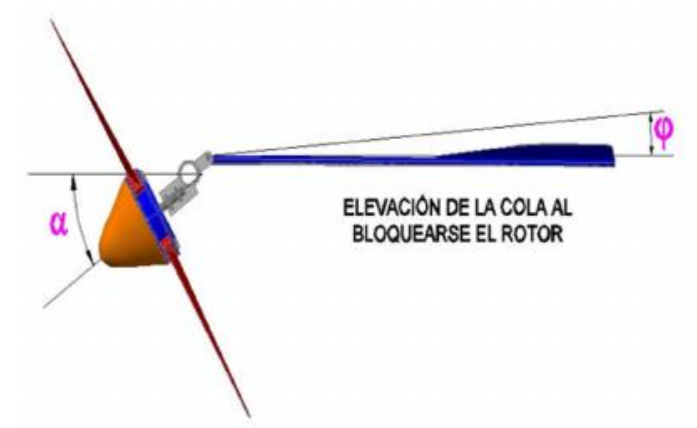

Fig. 5 Posición rotor-pivote de cola en la referencia III.

La matriz de cambio de referencia de II a III es:

$$
C_{I I-I I I}=\left[\begin{array}{ccc}
\cos \varphi & -\operatorname{sen} \varphi & 0 \\
\operatorname{sen} \varphi & \cos \varphi & 0 \\
0 & 0 & 1
\end{array}\right]
$$

\section{BALANCE DE FUERZAS Y MOMENTOS SOBRE LA COLA VELETA}

El análisis dinámico de fuerzas y momentos sólo del mecanismo cola-veleta, se realiza en la referencia III. Por tanto, en esta referencia se calcula la velocidad normal del viento en la veleta y el momento de inercia de la cola, ver fig. 6 .

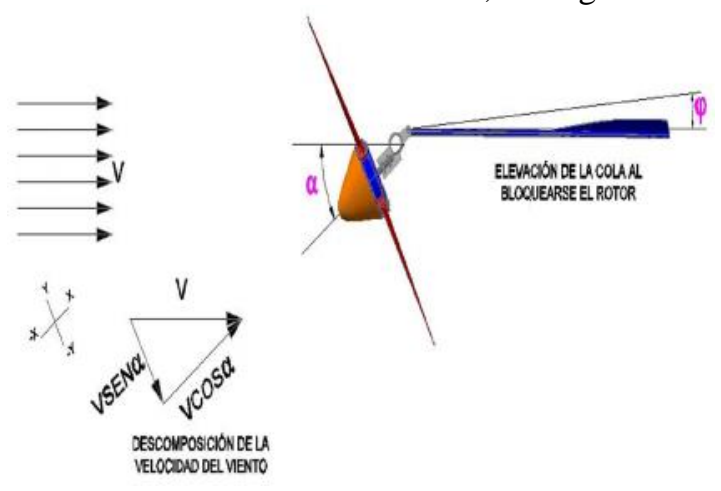

Fig. 6 Orientación cuando se da el bloqueo. 
El viento que incide sobre la veleta tiene un valor menor que el que incide en el rotor, es decir $V=$ $k . v$, donde $V$ es la velocidad del viento tras el rotor y $v$ es la velocidad del viento antes del rotor. Entonces la velocidad vectorial del viento en la cola será:

$$
\bar{V}^{0}=\left[\begin{array}{c}
V \cos \alpha \\
-V \operatorname{sen} \alpha \\
0
\end{array}\right]
$$

Para pasar el vector velocidad de la referencia 0 a la referencia III, se multiplica matricialmente a éste por las expresiones (1), (2) y (3), de donde se tiene que la componente de la velocidad normal del viento sobre la veleta es:

$\bar{V}_{y}^{\prime \prime \prime}=-V(-\cos \alpha \operatorname{sen} \varphi \cos \beta \cos \delta+$ $\cos \alpha \cos \varphi \operatorname{sen} \delta+\operatorname{sen} \alpha \operatorname{sen} \varphi \cos \beta \cos \delta$

$+\operatorname{sen} \alpha \cos \varphi \cos \delta$ )

Conociendo la componente " $y$ " de la velocidad y la componente " $y$ " del coeficiente de arrastre, la fuerza del viento sobre la veleta queda definida con la siguiente expresión:

$$
\bar{F}_{v, v e l, i}^{I I I}=\left\{\begin{array}{c}
0 \\
\frac{1}{2} \cdot \rho \cdot C_{D, y}^{I I I} \cdot S_{C} \cdot \bar{V}_{y}^{I I I} \cdot\left|\bar{V}_{y}^{I I I}\right| \\
0
\end{array}\right\}
$$

La fuerza vectorial que actúa en la cola, que es su peso con veleta incluida, se expresa como:

$$
\bar{F}_{w, C, i}^{\prime \prime \prime}=\left[\begin{array}{c}
m_{c} \cdot g \cos \varphi \operatorname{sen} \beta \\
m_{C} \cdot g \operatorname{sen} \varphi \operatorname{sen} \delta \\
-m_{C} \cdot g \cos \beta
\end{array}\right]
$$

Conocido las distancias de los puntos de aplicación de las fuerzas (empuje del viento a la veleta y peso de la cola) respecto al punto de rotación de la cola (pivote), se evalúa el momento de la cola en la referencia III que se efectúa sobre el eje de rotación (paralelo al eje $\mathrm{Z}^{\mathrm{III}}$ ), como el siguiente producto vectorial:

$$
\bar{M}_{C O L A}^{I I I}=\overline{O A}^{I I I} \times \bar{F}_{v, v e l, i}^{I I I}+\overline{O C}_{G C}^{I I I} \times \bar{F}_{w, C, i}^{I I I}
$$

Debido al giro libre sobre el eje $\mathrm{Z}_{\mathrm{III}}$, se debe imponer que la tercera componente de este momento, sea nula. De acá se logra encontrar una relación entre la velocidad del viento incidente $V$, el ángulo de bloqueo $\alpha$ y el ángulo de elevación de la cola $\varphi$. Entonces reemplazando se obtiene:

$$
\begin{aligned}
& V^{2}(-\cos \alpha \operatorname{sen} \varphi \cos \beta \cos \delta+\cos \alpha \cos \varphi \operatorname{sen} \delta \\
& +\operatorname{sen} \alpha \operatorname{sen} \varphi \cos \beta \cos \delta+\operatorname{sen} \alpha \cos \varphi \cos \delta)^{2} \\
& -\frac{m_{C} \cdot g \cdot L_{C G} \cdot \operatorname{sen} 2 \varphi \cdot \operatorname{sen} 2 \beta}{\rho \cdot C_{D, y}^{I I I} \cdot S_{C} \cdot L_{C} \cdot \cos \varphi \cos \beta}=0
\end{aligned}
$$

Agrupando constantes como:

$$
D=\rho \cdot C_{D, y}^{I I I} \cdot S_{C} \cdot L_{C} \cdot \cos \beta \cdot k^{2} \text { y } E=m_{C} \cdot g \cdot L_{C G} \cdot \operatorname{sen} 2 \beta
$$

se obtiene la primera ecuación generatriz (I):

$$
\begin{aligned}
& D \cdot \cos \varphi \cdot v^{2} \cdot(-\cos \alpha \operatorname{sen} \varphi \cos \beta \cos \delta \\
& +\cos \alpha \cos \varphi \operatorname{sen} \delta+\operatorname{sen} \alpha \operatorname{sen} \varphi \cos \beta \cos \delta \\
& +\operatorname{sen} \alpha \cos \varphi \cos \delta)^{2}-E \cdot \operatorname{sen} 2 \varphi=0
\end{aligned}
$$

\section{BALANCE DE FUERZAS Y MOMENTOS SOBRE EL SISTEMA COMPLETO}

En este caso se tiene en cuenta la cola veleta, el generador y la turbina, ello analizado sobre la referencia 0 . Acá se considera la acción del momento resultante de la cola en la referencia 0 . Cuando se hace el estudio al sistema de manera global, aparecen dos fuerzas correspondientes a la ocasionada por el viento en las palas y, al peso del aerogenerador (incluido turbina y generador). La componente tangencial de la fuerza del viento sobre las palas (fuerza torsional) se considera nula porque es pequeña comparada con la fuerza axial. Entonces queda sólo la fuerza axial:

$$
\bar{F}_{v, \text { rotor }}^{0}=\left\{\begin{array}{c}
\frac{1}{2} \rho \cdot C_{x} \cdot\left|V_{x}^{0}\right| \cdot V_{x}^{0} \cdot S \\
0 \\
0
\end{array}\right\}
$$

La otra fuerza que actúa es el peso del aerogenerador, exceptuando la cola y la torre, expresado como $\bar{F}_{w, a, i}^{0}=m_{A} \cdot g_{i}^{0}$ 
Conociendo las fuerzas de empuje del viento sobre las palas (fuerza axial), el peso del generador, la fuerza y el momento resultantes de la cola, se obtiene el momento total del sistema en la referencia 0 :

$$
\begin{aligned}
& \bar{M}_{\text {TOTAL }}^{0}=\bar{M}_{\text {COLA }}^{0}+\overline{X B} \times \bar{F}_{v, \text { rotor }}^{0}+ \\
& \overline{X C}_{G G} \times \bar{F}_{w, a}^{0}+\overline{X O} \times \bar{F}_{\text {COLA }}^{0}-\bar{M}_{f}
\end{aligned}
$$

Por el giro libre en el eje $Z_{0}$, esto es momento nulo, interesa sólo conocer el tercer componente vectorial, es decir:

$$
\begin{aligned}
& -\frac{1}{2} \cdot \rho \cdot C_{D, y}^{I I I} \cdot\left|V_{y}^{I I I}\right|^{2} \cdot S_{C} \cdot L_{C} \cdot \cos \varphi \cdot \cos 2 \beta \\
& +\frac{1}{2} \cdot \rho \cdot C_{x} \cdot\left|V_{x}^{0}\right|^{2} \cdot S \cdot e- \\
& \frac{1}{2} \cdot \rho \cdot C_{D, y}^{0} \cdot\left|V_{y}^{0}\right|^{2} \cdot S_{C} \cdot p-M_{f, z}=0
\end{aligned}
$$

Si para $v=v_{0}$, entonces $\alpha=0$ y $\varphi=0$; esto quiere decir que hasta una determinada velocidad de viento $\mathrm{v}_{\mathrm{o}}$ el rotor debe mantenerse frontal al viento con un ángulo $\alpha$ igual a cero.

Entonces de la ecuación 12 se obtiene lo siguiente:

$$
M_{f . z}=\frac{1}{2} \rho\left(e \cdot C_{x} v_{0}^{2} S-C_{D, y}^{I I I} \cdot v_{0}^{2} L_{C} S_{C} \operatorname{sen}^{2} \delta \cdot \cos 2 \beta\right)
$$

Tomando la componente z de la expresión 11 e igualando a cero, se tiene:

$$
\begin{aligned}
& -\frac{1}{2} \cdot \rho \cdot C_{D, y}^{I I I} \cdot\left|V_{y}^{I I}\right|^{2} \cdot S_{C} \cdot L_{C} \cdot \cos \varphi \cdot \cos 2 \beta+ \\
& \frac{1}{2} \cdot \rho \cdot C_{x} \cdot\left|V_{x}^{0}\right|^{2} \cdot S \cdot e-\frac{1}{2} \cdot \rho \cdot C_{D, y}^{0} \cdot\left|V_{y}^{0}\right|^{2} \cdot S_{C} \cdot p- \\
& \frac{1}{2} \cdot \rho\left(e C_{x} \cdot v_{0}^{2} \cdot S-C_{D, y}^{I I I} \cdot v_{0}^{2} L_{C} \cdot S_{C} \operatorname{sen}^{2} \delta \cdot \cos 2 \beta\right)=0
\end{aligned}
$$

Haciendo:

$$
\begin{aligned}
& A=2 \cdot L_{C} \cdot m_{C} \cdot g \cdot \operatorname{sen} \beta \cdot \cos 2 \beta / \rho \\
& B=e \cdot C_{x} \cdot S \\
& C=p \cdot C_{D, y}^{0} \cdot S \\
& R=e \cdot C_{x} \cdot v_{0}^{2} \cdot S-C_{D, y}^{\prime \prime \prime} \cdot v_{0}^{2} \cdot L_{C} \cdot S_{C} \cdot \operatorname{sen}^{2} \delta \cdot \cos 2 \beta
\end{aligned}
$$

Introduciendo las nuevas constantes y despejando la velocidad, se obtiene la segunda ecuación generatriz (II):

$$
v^{2}=\frac{R+A \cdot \operatorname{sen} 2 \varphi}{B \cdot \cos ^{2} \alpha-C \cdot \operatorname{sen}^{2} \alpha}
$$

Mediante estas dos ecuaciones generatrices (I) y (II), se logra las relaciones existentes entre la velocidad del viento $\mathrm{v}$, el ángulo de bloqueo $\alpha$ y el ángulo de elevación de la cola $\varphi$, para así dimensionar y diseñar de manera eficaz el dispositivo en estudio.

\section{CALCULO DE LA DINAMICA DEL SISTEMA}

Con la formulación anterior se procede al cálculo de todos los parámetros de diseño, para asî determinar la geometría del sistema de regulación y control de potencia en micro aerogeneración con mecanismo pivote de cola veleta. Para calcular el centro de gravedad de la cola se utiliza el análisis de cuerpos compuestos, de igual forma para el subsistema turbina-generador. En cuanto al área de la veleta se dimensiona entre 1/10 a 1/14 del área del rotor. Los cálculos y experimento se realizan para un pequeño prototipo aerogenerador de $50 \mathrm{~W}$ de $1.24 \mathrm{~m}$ de diámetro externo de rotor, acoplado a un generador eléctrico de imanes permanentes de Neodimio.

El tratamiento de la información de entrada y proceso de cálculo se ha realizado en el programa EXCEL. Como resultados se tiene la simulación del funcionamiento del sistema aerogenerador con pivote de cola veleta, donde a partir de la geometría, masas y resolución de las ecuaciones (I) y (II), se determina la variación teórica del control de potencia, a velocidades de viento que superan las condiciones nominales, para ello variando los ángulos $\alpha$ y $\varphi$. Así se logra determinar inicialmente cuánto se bloquea (valor de $\alpha$ ) el rotor a una determinada velocidad de viento (v), y cuanto se eleva la cola con respecto a su posición original (valor de $\varphi$ ).

A partir de estos resultados se diseña y construye el dispositivo pivote de pruebas, para ser ensayado en el Túnel de Viento y determinar su geometría final.

Tabla 1. Dinámica del sistema y cálculo de constantes de las ecuaciones (I) y (II). 


\begin{tabular}{llll}
\hline \multicolumn{1}{c}{ NOMBRE } & SÍMBOLO & DATOS & UNIDAD \\
\hline Masa de la cola & $\mathrm{m}_{\mathrm{C}}=$ & 5,24 & $\mathrm{~kg}$ \\
Longitud de O al centro de gravedad $\left(\mathrm{C}_{\mathrm{GC}}\right)$ & $\mathrm{L}_{\mathrm{CG}}=$ & 0,53 & $\mathrm{~m}$ \\
Longitud de OA & $\mathrm{L}_{\mathrm{C}}=$ & 1,20 & $\mathrm{~m}$ \\
Superficie total de la veleta de la cola & $\mathrm{S}_{\mathrm{V}}=$ & 0,17 & $\mathrm{~m}^{2}$ \\
Superficie abarcada por el rotor eólico & $\mathrm{S}=$ & 1,21 & $\mathrm{~m}^{2}$ \\
Ángulo vertical entre el eje del rotor y el pivote o bisagra & $\beta=$ & 8 & $\circ$ \\
Gravedad & $\mathrm{g}=$ & 9,81 & $\mathrm{~m} / \mathrm{s}$ \\
Densidad del aire & $\rho=$ & 1,205 & $\mathrm{~kg} / \mathrm{m}^{3}$ \\
Coeficiente de resistencia de la fuerza normal en la ref III & $\mathrm{C}^{3}{ }_{\mathrm{D} 2}=$ & 1,10 & \\
Coeficiente de empuje del viento sobre las palas & $\mathrm{C}^{0}{ }_{\mathrm{d}, 1}=$ & 0,89 & \\
Coeficiente de resistencia de la fuerza normal en ref 0 & $\mathrm{C}_{\mathrm{D}, 2}=$ & 1,10 & \\
Excentricidad del eje del rotor respecto del eje del mástil & $\mathrm{e}=$ & 0,0400 & $\mathrm{~m}$ \\
Distancia de XO en la componente x & $\mathrm{p}=$ & 0,13 & $\mathrm{~m}$ \\
Desviación de la veleta con respecto al eje horizontal & $\delta_{0}=$ & 6 & $\circ$ \\
Reducción de la velocidad & $\mathrm{k}=$ & 0,67 & \\
Ángulo relativo entre la dirección del viento y el eje & & & $\circ$ \\
normal del rotor eólico & $\alpha$ & & $\circ$ \\
Ángulo relativo entre el eje del rotor y la cola & $\varphi$ & & $\circ$ \\
Ángulo relativo entre la veleta y el viento & $\psi$ & & $\circ$ \\
Ángulo horizontal entre el eje del rotor y el pivote & $\mathrm{o}$ & & \\
bisagra (Desfase) & $\delta=$ & 45 & $\circ$ \\
Coeficiente de potencia & $\mathrm{C}_{\mathrm{P}}=$ & 0,35 & \\
Eficiencia del generador & $\eta=$ & 0,75 & \\
Velocidad específica de diseño (3 palas) & $\lambda_{\mathrm{d}}=$ & 6 & \\
Velocidad de viento donde vence el rozamiento & $\mathrm{v}_{0}=$ & 10 & $\mathrm{~m} / \mathrm{s}$ \\
Constante1 & $\mathrm{A}=$ & 13,70 & $\mathrm{~m} / \mathrm{s}^{2}$ \\
Constante2 & $\mathrm{B}=$ & 0,04 & $\mathrm{~m} \mathrm{~m}^{3}$ \\
Constante3 & $\mathrm{C}=$ & 0,17 & $\mathrm{~m} \mathrm{~m}^{3}$ \\
Rozamiento & $\mathrm{R}=$ & 4,06 & $\mathrm{~m}^{5} / \mathrm{s}^{2}$ \\
Constante4 & $\mathrm{D}=$ & 0,12 & $\mathrm{Kg}$ \\
Constante5 & $\mathrm{E}=$ & 7,56 & $\mathrm{Nxm}$ \\
\hline & & & \\
\hline
\end{tabular}

\section{DISEÑO, CONSTRUCCION Y PRUEBAS EXPERIMENTALES DE FUNCIONAMIENTO}

\section{Diseño y construcción}

En base al análisis realizado en el rubro anterior, se procede al diseño y construcción del dispositivo pivote de cola. Para ello, si bien se parte de las variables calculadas $\mathrm{v}, \alpha$ y $\varphi$, que explican el comportamiento dinámico tanto de la cola-veleta como del sistema aerogenerador como un todo, se toma la conveniencia de elaborar dos arreglos geométricos del dispositivo en estudio, esto es: una construcción del pivote de cola para pruebas y, luego la construcción del pivote de cola final. Así se procede a elaborar alternativas geométricas en estudio utilizando el programa AUTOCAD.
Pivote de cola para pruebas.- $\mathrm{Se}$ requiere conformar un arreglo donde se pueda variar (ajustar) en un rango de posibilidades: el ángulo pivote de cola, el ángulo del eje de la cola respecto a la dirección del viento $\mathrm{y}$, la excentricidad regulable sobre una tornamesa donde se pueda variar la posición del eje rotor con respecto al eje del poste.

Estos ajustes permiten una mayor maniobrabilidad y versatilidad de posiciones en las pruebas experimentales, cuyos resultados se visualizan en las figuras 7,8 y 9 : 


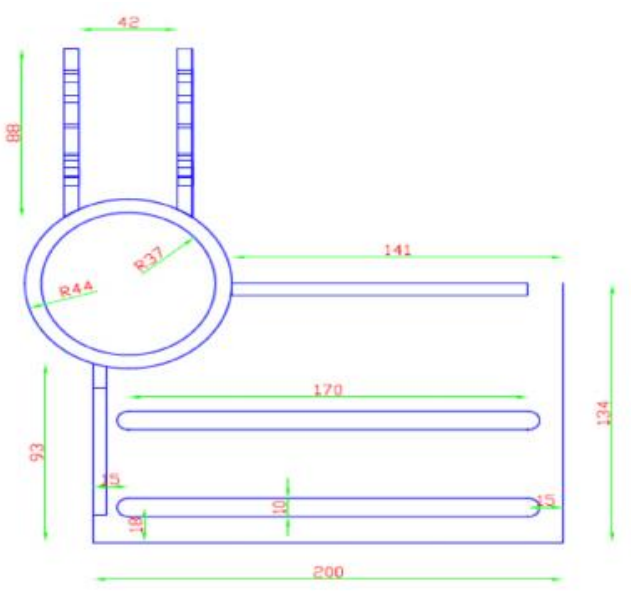

Fig. 7 Vista de planta y dimensionado del dispositivo pivote de cola para pruebas.

Pivote de cola final.- Una vez fijados el ángulo pivote de cola, ángulo de eje de cola y la excentricidad entre el eje rotor y eje de poste, todo ello determinados en las pruebas experimentales en el Túnel de Viento, se diseña y construye el elemento prototipo final.

Los resultados se muestran a continuación:

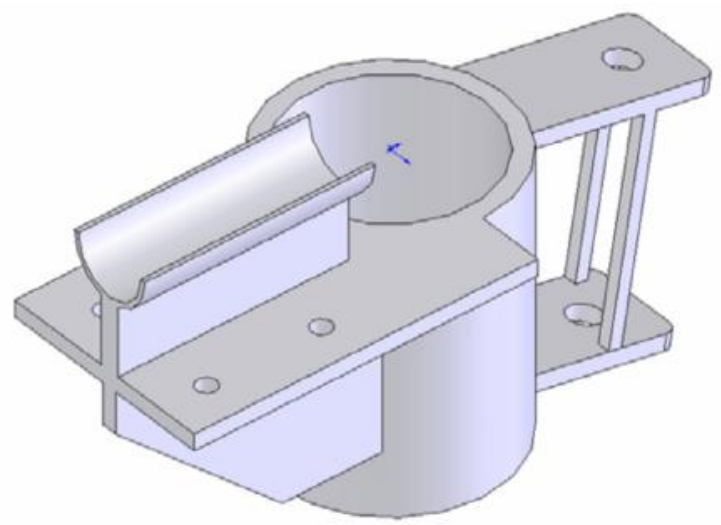

Fig. 8 Dispositivo pivote de cola final o definitivo.
Fig. 9 Dimensionado de planta del dispositivo

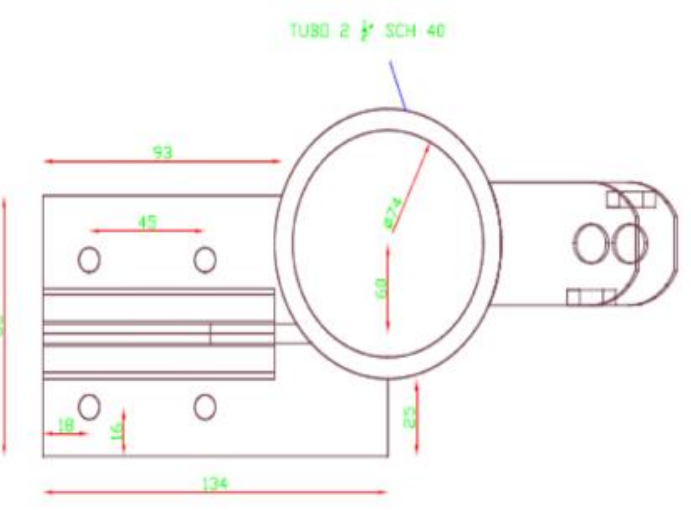

Pivote de cola final.

\section{PRUEBAS EXPERIMENTALES EN EL TUNEL DE VIENTO}

Construido el dispositivo pivote de cola para pruebas, se procede a los ensayos experimentales de funcionamiento en el Túnel de Viento. El objetivo es buscar que el aerogenerador en prueba comience a bloquearse (salirse fuera del frente de viento) a partir de una velocidad de viento superior a $10 \mathrm{~m} / \mathrm{s}$, manteniendo la potencia de generación no creciente, no obstante aumente la velocidad del viento.

El equipamiento lo constituye:

- Túnel de Viento del Laboratorio de Energía de la UNI. Se realizaron ajustes y pruebas de estabilidad de flujo para todo el rango de velocidades de viento simuladas.

- El aerogenerador modelo IT 50, instalado con generador de imanes permanentes de Neodimio.

- El dispositivo Pivote de Cola y veleta para pruebas, fig. 11.

La instrumentación está conformada por:

- Un variador de frecuencia, para la simulación de las velocidades de viento en el Túnel, ubicado en el tablero de mando del ventilador simulador de viento, fig. 12 .

- Un banco de resistencias para la disipación de la energía eléctrica generada, fijado al panel o tablero de control y mediciones.

- Voltímetros en AC y DC, fijado al panel o tablero de control y mediciones. 
- Amperímetros y/o pinza amperimétrica digital, ubicados en el panel de control y mediciones.

- Multitester digital.

- Un anemómetro de medición de la velocidad del viento, fijado a la entrada aguas arriba del aerogenerador.

- Una veleta de medición de giro, fijada a la entrada aguas abajo del aerogenerador.

- Un Data Logger para los registros de velocidad de viento en el anemómetro y la veleta de medición.

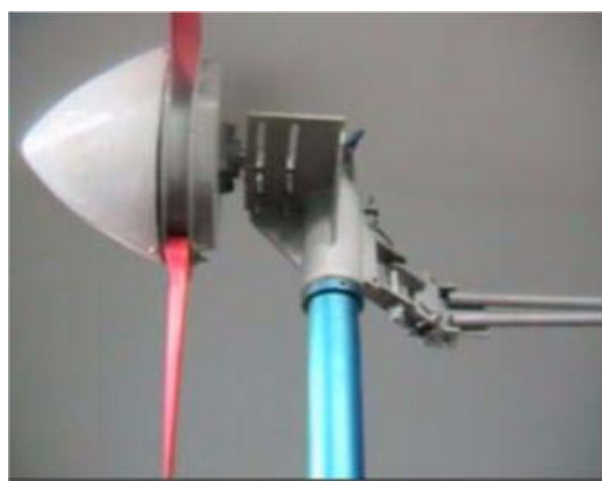

Fig. 10 Foto acople aerogenerador mecanismo pivote de cola para pruebas.

La distribución de los instrumentos en el panel o tablero de control, se muestra en la figura 11.

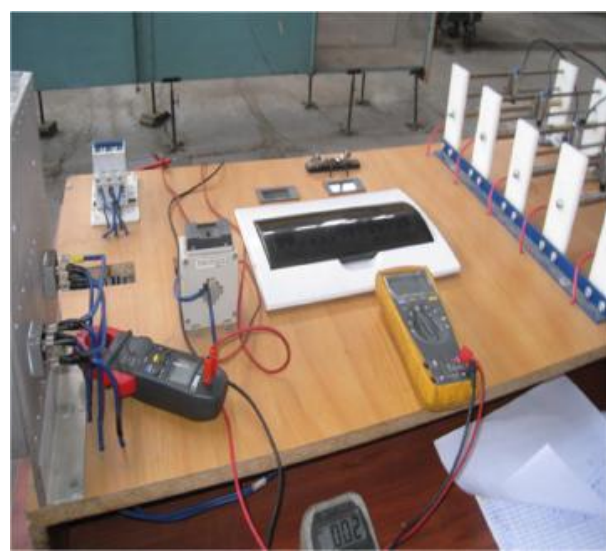

Fig.11 Tablero de control y mediciones.

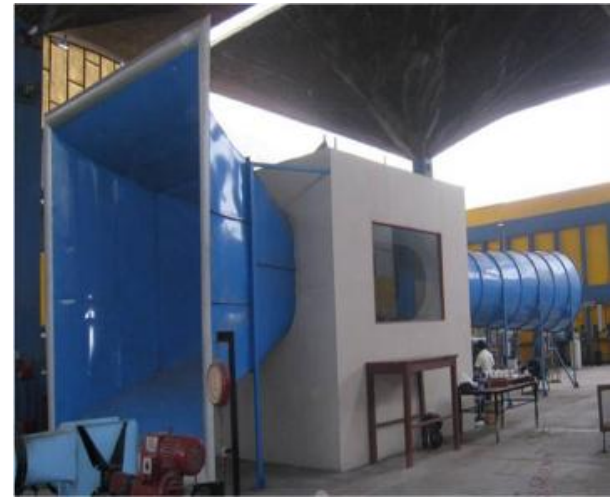

Fig. 12 Disposición del túnel de viento y tablero de control externo.

\section{ANALISIS DE RESULTADOS}

Los resultados experimentales que demuestran que el dispositivo pivote de cola responde a las características de diseño, se obtienen mediante las características de comportamiento del sistema aerogenerador en prueba; es decir, las variaciones de potencia generada en función de la velocidad y, las variaciones del ángulo de ataque frente a la velocidad de viento simulado. Así, la curva de potencia generada versus velocidad simulada del viento del aerogenerador con el dispositivo pivote de cola instalado, se muestra en la figura 13. Tal como se demuestra, a velocidades de viento superiores a las condiciones de trabajo del sistema, se logra controlar el crecimiento de la potencia generada en el rango superior a los $10 \mathrm{~m} / \mathrm{s}$. Para alcanzar este rango de control de potencia aerogeneradora, el dispositivo pivote de cola quedó ajustado a las siguientes características:

- Excentricidad entre eje rotor

- y eje de poste, e:

$6 \mathrm{~cm}$

- Angulo de pivote de cola, $\beta: 8^{\circ}$

- Angulo de eje de cola, $\delta$ :

$6^{\circ}$

Condiciones ambientales en el Túnel de Viento:

- Presión local:

$757 \mathrm{~mm} \mathrm{Hg}$

- Temperatura ambiente: $\quad 20^{\circ} \mathrm{C}$

En la figura 14 se ilustra la curva de potencia generada versus velocidad del viento del sistema aerogenerador sin el dispositivo pivote de cola, donde se observa que la potencia se incrementa sin control a medida que aumenta la velocidad del 
viento. En la figura 15, se presenta la variación del ángulo de bloqueo $\alpha$ en función de la velocidad de viento.

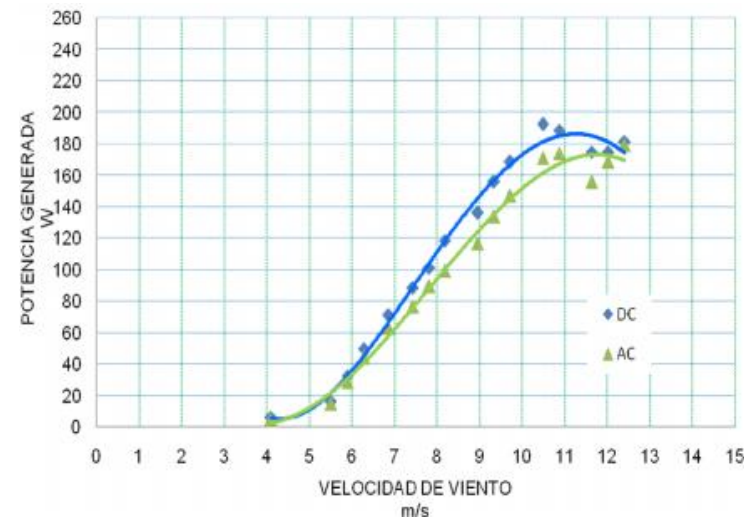

Fig. 13 Curva de potencia con dispositivo pivote de cola.

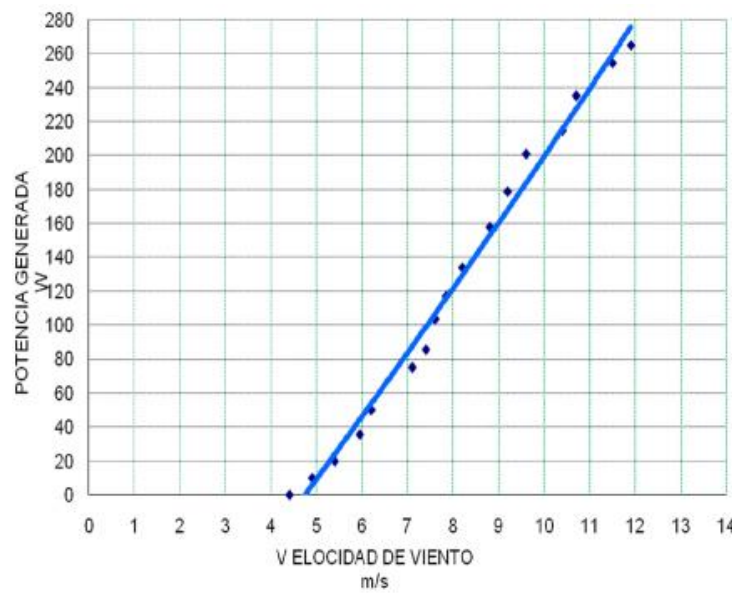

Fig. 14 Curva de potencia sin dispositivo pivote de cola.

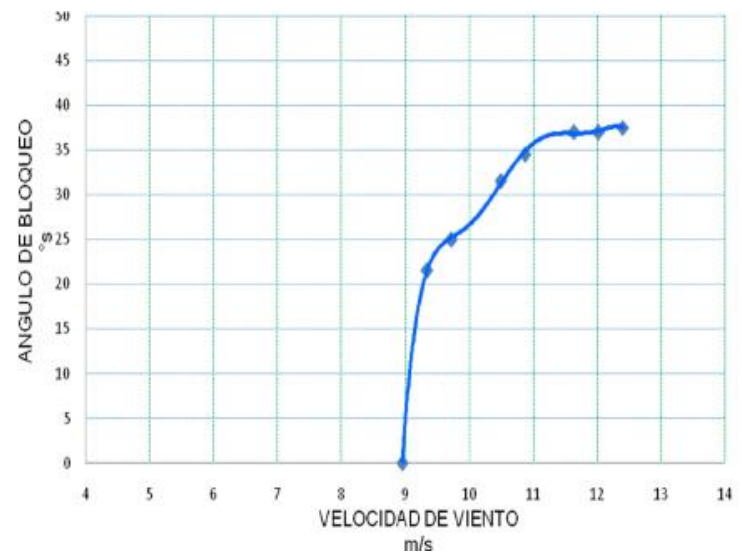

Fig. 15 Variación del ángulo de bloqueo con la velocidad de viento extremo.

\section{CONCLUSIONES}

De los ensayos experimentales en el Túnel de Viento, con un microaerogenerador de pruebas, se demuestra que para una excentricidad de $6 \mathrm{~cm}$, un ángulo $\beta$ de $8^{\circ}$ y un ángulo $\delta$ de $6^{\circ}$, se logra diseñar el mejor dispositivo mecánico que regula y controla la potencia instantánea en sobrevelocidades de viento por encima de los $10 \mathrm{~m} / \mathrm{s}$.

En las pruebas realizadas con el mismo aerogenerador, pero prescindiendo del dispositivo pivote de cola, la curva de potencia del aerogenerador se incrementa de manera descontrolada con el aumento de velocidad, situación que a velocidades superiores a los 14 $\mathrm{m} / \mathrm{s}$, el generador de imanes permanentes utilizado comienza a sobrecalentarse de forma peligrosa.

El ángulo de bloqueo $\alpha$, experimentalmente muestra su crecimiento arriba de $9 \mathrm{~m} / \mathrm{s}$ de velocidad de viento simulado, ello significa el inicio del control de la potencia de generación, es decir sacar a la turbina-generador fuera del frente de viento a velocidades superiores a las condiciones a que fue diseñado el sistema aerogenerador.

El éxito del buen diseño del dispositivo pivote de cola, se inicia en el cálculo de la dinámica de funcionamiento del conjunto turbina-generador / pivote de cola-veleta, obteniendo las ecuaciones generatrices (I) y (II), y que se consolida su construcción definitiva gracias a las pruebas experimentales en el Túnel de Viento.

La ventaja de los ensayos del dispositivo en el Túnel de Viento, comparado a las pruebas que se harían en campo, es enorme, pues permite hacer verificaciones y fijaciones de los ángulos de diseño a velocidades de viento controladas.

Los resultados obtenidos en el aerogenerador en prueba IT50, pueden ser extendidos a los parámetros de diseño e, $\beta$ y $\delta$ correspondientes a pequeños aerogeneradores de otras capacidades, y a partir de ello estimar sus respectivas curvas de potencia de diseño y potencias límite controladas.

\section{REFERENCIAS}

1. Gonzáles Chávez, S., Chiroque Baldera J. "Diseño y construcción de un aerogenerador 
de $500 \mathrm{~W}$ con imanes permanentes para pequeñas demandas eléctricas de zonas rurales". TECNIA, UNI, Vol. 18, N², 2008.

2. Gonzáles Chávez, S., "Aerogeneración, una Fuente Energética Competitiva", Reg. ISBN 8492334533, España, 2000.

3. Manwell, J. F., McGowan, J. G., Rogers A. L., "Wind Energy Explained. Theory, Design and Application". Ed. Wiley, 2002.

4. ITDG Soluciones Prácticas, "Small Wind Systems for Battery Charging", Documentos internos, Lima, 2000.
5. Rodríguez Amenedo, J. L., Burgos Díaz, J. C., Arnaltes Gómez, S., "Sistemas Eólicos de Producción de Energía Eléctrica". Editorial Rueda S. L., 2003.

6. Arribas, L., "Microaerogeneración y Pequeños Aerogeneradores, CIEMAT, España, 2007.

Correspondencia: salome@uni.edu.pe

Recepción de originales: setiembre 2012

Aceptación de originales: enero 2013 